Research Paper

\title{
Genomic Profiling Reveals the Potential Role of TCLIA and MDRI Deficiency in Chemotherapy-Induced Cardiotoxicity
}

Timothy A. McCaffrey 1,6, Constantine Tziros², Jannet Lewis², Richard Katz², Robert Siegel ${ }^{3}$, William Weglicki ${ }^{2,4}$, Jay Kramer ${ }^{4}$, I. Tong Mak', Ian Toma ${ }^{1}$, Liang Chen ${ }^{1}$, Elizabeth Benas ${ }^{1}$, Alexander Lowitt ${ }^{1}$, Shruti Rao' ${ }^{1}$, Linda Witkin², Yi Lian'1, Yinglei Lai ${ }^{5}$, Zhaoqing Yang ${ }^{1}$ and Sidney W. Fu ${ }^{1,6}{ }^{\bowtie}$

1. Department of Medicine, Division of Genomic Medicine;

2. Department of Medicine, Division of Cardiology;

3. Department of Medicine, Division of Hematology/Oncology;

4. Department of Biochemistry and Molecular Biology;

5. Department of Statistics and Biostatistics Center;

6. Department of Microbiology, Immunology and Tropical Medicine, The GW Heart and Vascular Institute, and The Dr. Cyrus and Myrtle Katzen Cancer Center, George Washington University School of Medicine and Health Sciences, Washington DC, USA.

$\square$ Corresponding author: Sidney W. Fu, M.D., Ph.D. Department of Medicine, Division of Genomic Medicine, The George Washington University School of Medicine and Health Sciences, 2300 Eye Street, N.W. Ross Hall 402C, Washington, DC 20037, Email: sfu@gwu.edu Tel: 202-994-4767 Fax: 202-994-8924.

(c) Ivyspring International Publisher. This is an open-access article distributed under the terms of the Creative Commons License (http://creativecommons.org/ licenses/by-nc-nd/3.0/). Reproduction is permitted for personal, noncommercial use, provided that the article is in whole, unmodified, and properly cited.

Received: 2013.02.09; Accepted: 2013.02.27; Published: 2013.04.22

\begin{abstract}
Background: Anthracyclines, such as doxorubicin (Adriamycin), are highly effective chemotherapeutic agents, but are well known to cause myocardial dysfunction and life-threatening congestive heart failure (CHF) in some patients.

Methods: To generate new hypotheses about its etiology, genome-wide transcript analysis was performed on whole blood RNA from women that received doxorubicin-based chemotherapy and either did, or did not develop CHF, as defined by ejection fractions (EF) $\leq 40 \%$. Women with non-ischemic cardiomyopathy unrelated to chemotherapy were compared to breast cancer patients prior to chemo with normal EF to identify heart failure-related transcripts in women not receiving chemotherapy. Byproducts of oxidative stress in plasma were measured in a subset of patients.

Results: The results indicate that patients treated with doxorubicin showed sustained elevations in oxidative byproducts in plasma. At the RNA level, women who exhibited low EFs after chemotherapy had 260 transcripts that differed $>2$-fold $(p<0.05)$ compared to women who received chemo but maintained normal EFs. Most of these transcripts (20I) were not altered in non-chemotherapy patients with low EFs. Pathway analysis of the differentially expressed genes indicated enrichment in apoptosis-related transcripts. Notably, women with chemo-induced low EFs had a 4.8-fold decrease in T-cell leukemia/lymphoma IA (TCLIA) transcripts. TCLIA is expressed in both cardiac and skeletal muscle, and is a known co-activator for AKT, one of the major pro-survival factors for cardiomyocytes. Further, women who developed low EFs had a 2-fold lower level of $A B C B I$ transcript, encoding the multidrug resistance protein I (MDRI), which is an efflux pump for doxorubicin, potentially leading to higher cardiac levels of drug. In vitro studies confirmed that inhibition of MDRI by verapamil in rat $\mathrm{H} 9 \mathrm{C} 2$ cardiomyocytes increased their susceptibility to doxorubicin-induced toxicity.
\end{abstract}


Conclusions: It is proposed that chemo-induced cardiomyopathy may be due to a reduction in TCLIA levels, thereby causing increased apoptotic sensitivity, and leading to reduced cardiac MDRI levels, causing higher cardiac levels of doxorubicin and intracellular free radicals. If so, screening for TCLIA and MDRI SNPs or expression level in blood, might identify women at greatest risk of chemo-induced heart failure.

Key words: doxorubicin, adriamycin, heart failure, cardiomyopathy, microarray, expression profiling, free radicals, multidrug resistance protein, MDR1, TCL1A.

\section{Introduction}

Anthracyclines, such as doxorubicin (Adriamycin), have been key cancer chemotherapeutic agents for the past 30 years. Despite their demonstrated benefit in cancer chemotherapy, anthracyclines can cause myocardial dysfunction in up to $25 \%$ of patients and life threatening heart failure in $1-4 \%$ of patients [1]. Cardiotoxicity due to doxorubicin is dose-related, with doses exceeding $500 \mathrm{mg} / \mathrm{kg}$ associated with the greatest incidence of left ventricular (LV) dysfunction and development of clinically overt congestive heart failure (CHF), often manifest as a reduction in the left ventricular ejection fraction (EF) [2]. However, patients receiving lower doses of doxorubicin also develop evidence of cardiotoxicity, and a wide individual susceptibility to cardiac damage has been observed.

Anthracyclines attack tumor cells by intercalating on DNA, inhibiting DNA replication, and thereby generating DNA damage, thus triggering apoptosis. Rapidly growing tumor cells are more susceptible than non-dividing cells, such as cardiomyocytes and neurons, thus conferring a relatively selective anti-tumor effect. However, it is unclear why some women are especially sensitive to the toxic effects of anthracyclines on cardiomyocytes. The mechanism by which anthracyclines produce myocardial damage is thought to involve a marked increase in oxidative stress, culminating in cardiomyocyte apoptosis. There appears to be a subset of individuals who are more susceptible to drug-induced oxidative damage and apoptosis. This susceptibility appears to be exacerbated with the use of trastuzumab, a monoclonal antibody to the HER2 proto-oncogene, a member of epidermal growth factor (EGF) receptor family. The addition of trastuzumab to anthracycline therapy has been shown to improve the chemotherapeutic response, but is also associated with a marked increase in the development of CHF [3], with the incidence of CHF increasing from $8 \%$ to $27 \%$ when trastuzumab was added [4]. Thus, the identification of women prone to CHF after chemotherapy is key to tipping the balance between effective chemotherapy and potentially life-threatening adverse effects.
A growing literature indicates that changes in gene expression of circulating cells can reflect changes in other organ systems. Published studies have documented mRNA expression changes in blood in disease states such as vasculitis [5], lupus [6], and coronary artery disease $[7 ; 8]$. The potential of this methodology in heart failure research has recently been reviewed by Liew [9]. Thus, the present studies were designed to identify mRNA expression markers in patient's blood and to determine whether any of the markers might elucidate possible mechanisms of enhanced susceptibility to anthracycline-induced cardiotoxicity. Minimally, biomarkers of chemo-induced CHF could allow individualization of chemotherapy, and importantly, administration of potentially preventive medications such as angiotensin receptor blockers, anti-oxidants, and beta-blockers.

\section{Methods}

\section{Subjects}

Patients at The George Washington University Medical Faculty Associates presenting for treatment of cancer using anthracyclines as part of a chemotherapeutic protocol, with or without adjuvant trastuzumab, were enrolled in an Institutional Review Board-approved protocol and gave informed consent.

\section{Inclusion/Exclusion Criteria}

All women presenting for anthracycline-based chemotherapy were considered eligible. Patients were excluded if their pre-chemo evaluation indicated abnormal left ventricular systolic function (ejection fraction $<50 \%$ ), past history of, or active cardiac disease including: myocardial infarction, congestive heart failure, angina pectoris requiring medication, arrhythmia requiring medication, clinically significant valvular disease (i.e., valvular stenosis of any degree, regurgitation greater than mild in severity), or uncontrolled systemic hypertension.

\section{Echocardiography}

A standard two-dimensional and Doppler study, including tissue Doppler imaging, was performed at 
baseline, prior to receiving chemotherapy. Left ventricular systolic function was assessed using biplane Simpson's method for calculation of ventricular volumes and EF. Echocardiograms were repeated after completion of the chemotherapy protocol, or if the patient developed clinical symptoms of heart failure.

\section{Assessment of LV Diastolic Function}

Diastolic function was evaluated by pulsed and tissue Doppler for estimation of pulmonary capillary wedge pressure (PCWP) using the formula (PCWP= $1.24[\mathrm{E} / \mathrm{Ea}]+1.9)[10]$, and color tissue Doppler assessment for diastolic dyssynchrony [11]. Echocardiography and pre-defined parameters were repeated immediately after the final cycle of anthracyclines (typically 8 weeks), 6 months and 12 months. Patients receiving additional chemotherapy with trastuzumab also underwent clinically indicated echocardiograms prior to initiation of therapy, then at 6 months and 12 months.

\section{Markers of Oxidative Stress}

Blood samples were obtained prior to chemotherapy, and 24 hours following completion of each infusion cycle. Accumulation of lipid peroxidation products in the blood were assessed semi-quantitatively by the measurement of thiobarbituric-acid reactive substances (TBARS), as described [12]. Briefly, fresh plasma was acidified with trichloroacetic acid to prevent post-isolation changes and then reacted with 2-thiobarbituric acid and butylated hydroxytoluene at $80^{\circ} \mathrm{C}$ for $30 \mathrm{~min}$, and the chromophore developed in the supernatant was measured at $532 \mathrm{~nm}$.

\section{Blood RNA Isolation}

Blood was collected by venipuncture directly into PaxGene Blood RNA collection tubes (PreAnalytiX). The blood was incubated 2 hours at $25^{\circ} \mathrm{C}$ to complete the cell lysis and RNAse neutralization, moved to $-20^{\circ} \mathrm{C}$ overnight, and then stored at $-80^{\circ} \mathrm{C}$ until processing. Whole blood RNA was isolated using the PaxGene blood RNA isolation system with on-column DNAse treatment. RNA samples were further purified for microarray analysis using RNAeasy Mini kit (Qiagen). RNA concentration was quantitated by absorbance at $260 \mathrm{~nm}$ (Nanodrop), and the RNA integrity was tested by capillary electrophoresis on an Agilent 2100 Bioanalyzer. The typical yield of RNA was $\sim 7 \mu \mathrm{g} / 2.5 \mathrm{ml}$ whole blood.

\section{Microarray Data Analysis}

Purified RNA (100 ng) was reverse transcribed with SuperScript III using random hexamers and the
cDNA was amplified with Ovation RNA Amplification System V2 kit (NuGEN). The cDNA was further purified with DNA Clean \& Concentrator-25 (Zymo Research), and then $3.75 \mu \mathrm{g}$ of cDNA was fragmented and labeled with FL-Ovation cDNA Biotin Module V2 kit (NuGEN) and hybridized to an Affymetrix GeneChip Human Genome U133 Plus 2.0 Array for 18 hours. The hybridization of fluorescently labeled cDNA was quantitated on an Affymetrix GeneChip Scanner 3000 7G.

\section{Statistical Methods}

The microarray probeset levels were converted to transcript levels using RMA summarization in GeneSpring GX 10 (Agilent Technologies). To identify transcripts associated with chemo-induced $\mathrm{CHF}$, the 54,000 total transcripts were analyzed by an unpaired t-test ( $p$-value cut-off $\leq 0.05$ ), and further filtered for a fold-change of $>1.5$ between groups. To understand the possible relationships between these transcripts, the differentially expressed gene (DEG) list was imported into Ingenuity Pathway Analysis (IPA) software. Functional annotation and gene ontology of DEGs was further explored with DAVID [13].

\section{Genotyping Analysis}

DNA from subjects was purified from Paxgene preserved whole blood by centrifugation of $500 \mu \mathrm{l}$ and treating the pellet with RNAse/proteinase $\mathrm{K}$ followed by phenol/chloroform extraction and ethanol precipitation [14]. SNPs in TCL1A were determined by TaqMan assay for the rs 11849538 SNP on an ABI 7300, with dissociation temperature of $95^{\circ} \mathrm{C}$ and annealing temperature of $60^{\circ} \mathrm{C}$. From the 24 patients for which expression data was obtained, sufficient DNA and genotyping results were obtained from 18 of the patients, including all 5 patients in the chemo-induced low EF group.

\section{Cell culture}

Rat cardiomyocyte progenitors (H9C2) were obtained from American Type Culture Collection (ATCC) and maintained in DMEM with 10\% FBS and antibiotics. The MDR1 inhibitor, verapamil (Sigma), was tested by plating cells at 50,000 cells/well of a 24 well plate and allowing them to adhere overnight. Verapamil was added 2 hours prior to the addition of doxorubicin at specified doses. After 20 hours with doxorubicin, the viable cells were quantitated by their ability to metabolize MTT as described [15]. Briefly, the culture media was replaced with MTT for 4 hours and the metabolic product was quantified by dissolving the cells in $200 \mu \mathrm{l}$ of DMSO and transferring the media to a 96 well plate, where absorbance at 570 
$\mathrm{nm}$ was determined in a plate spectrophotometer.

\section{Results}

To generate new hypotheses about the etiology of chemo-induced $\mathrm{CHF}$, we performed genome-wide transcript analysis with the Affymetrix U133+2 GeneChip on whole blood RNA from women receiving chemotherapy with anthracyclines who either did (Group A, n=5), or did not (Group B, n=10) develop subsequent cardiac dysfunction, as defined by ejection fractions $(\mathrm{EF}) \leq 40 \%$. Women with non-ischemic cardiomyopathy not due to chemotherapy (Group C, $\mathrm{n}=4$ ) were compared to breast cancer patients prior to chemo with normal EF (Group D, n=5) to identify heart failure-related transcripts independent of chemotherapy. The general experimental design is summarized in Figure 1.

\section{Clinical Parameters}

Demographic and baseline cardiovascular parameters are shown in Table 1 . While increasing age has been reported to be associated with reduced LV performance after chemotherapy [16], there was no significant difference in age between women who did or did not develop HF after chemo in our cohort $(p>0.05)$. There were more women in Group D with hypertension and diabetes, but with the small group size, the difference was not statistically significant.

A comparison of the two-dimensional echo images of a patient with normal LV systolic function versus a patient with chemo-induced dilated cardiomyopathy is shown in Additional file 1: Supplemen- tary Figure 1. In the patient with normal LV function, the heart size is normal in diastole and decrease normally in systole, reflecting a normal EF. In contrast, with chemo-induced cardiomyopathy, the heart is dilated and shows little change in size with systolic contraction, and thus, in these patients, EF is typically significantly reduced.

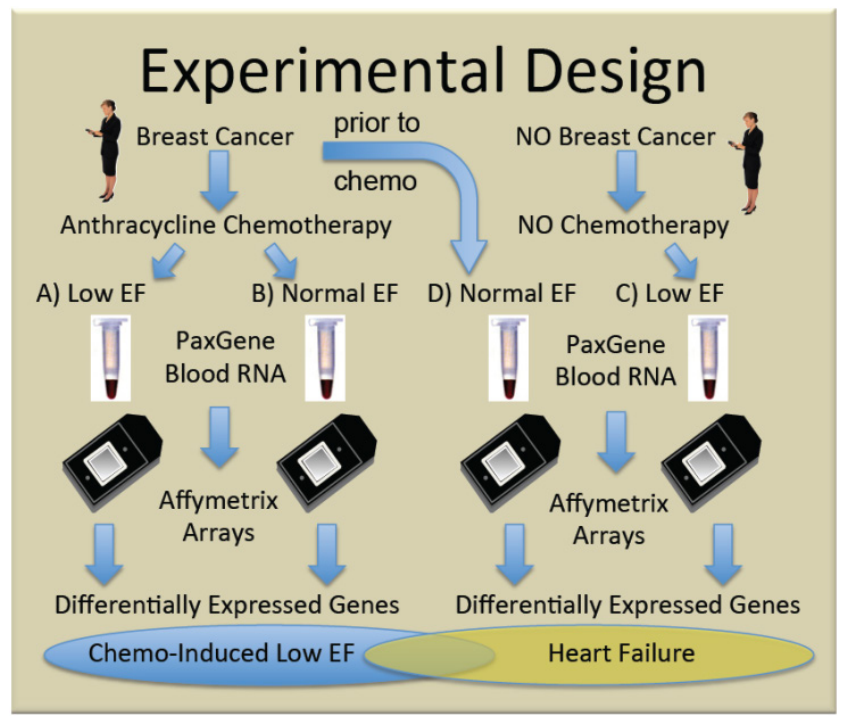

Fig I. Experimental design. Women with, and without breast cancer gave informed consent and were enrolled in the study. Some women with breast cancer given doxorubicin-containing chemotherapy developed clinically significant low ejection fractions (EF) (Group A) and were compared to similar women who did not develop low EF (Group B). To identify transcripts correlating with CHF, independent of chemotherapy, women with breast cancer and normal ejection fractions, were sampled prior to chemotherapy (Group D), and compared with women who developed low EFs but never received chemotherapy (Group C).

Table I. Classification and Baseline Values of Patients.

\begin{tabular}{|c|c|c|c|c|c|}
\hline Group & A & B & $\mathrm{C}$ & $\mathrm{D}$ & \\
\hline Name & BrCa-Chemo-Low EF & BrCa-Chemo-Norm EF & No Ca-NoChemo-Low EF & BrCa-preChemo-Norm EF & \\
\hline $\mathrm{N}=$ & 5 & 10 & 4 & 5 & \\
\hline Breast Cancer & Yes & Yes & No & Yes & \\
\hline Chemotherapy & Yes & Yes & No & No & \\
\hline Low Ejection Fraction & Yes & No & Yes & No & \\
\hline Age (mean) & 59.2 & 58.3 & 53.4 & 48.1 & NS \\
\hline Age (median, range) & $56(51-80)$ & $60(42-76)$ & $51(47-63)$ & $53(43-58)$ & \\
\hline Ejection Fraction (EF) & 24.8 & 60.9 & 26.6 & 68.0 & $\mathrm{p}<.001$ \\
\hline EF (median, range) & $20(15-40)$ & $58(52-69)$ & $24(22-35)$ & $66(55-86)$ & \\
\hline Hypertensive & $0 \%$ & $0 \%$ & $0 \%$ & $40 \%$ & NS \\
\hline Diabetic & $0 \%$ & $0 \%$ & $0 \%$ & $20 \%$ & NS \\
\hline
\end{tabular}


Table 2. Selected differentially expressed transcripts in chemo-induced low ejection fractions.

\begin{tabular}{|c|c|c|}
\hline Gene ID & Fold Change Gene & Description \\
\hline \multicolumn{3}{|c|}{ Apoptosis/Survival Signalling } \\
\hline 330 & 2.02 down BIRC3 & IAP homolog C (MIHC) \\
\hline 9994 & 2.58 down CASP8AP2 & CASP8 associated protein 2 \\
\hline 958 & 2.61 down $\mathrm{CD} 40$ & NGF receptor-related B-cell activation \\
\hline 5074 & 2.00 down PAWR & PRKC, apoptosis, WT1, (PAWR) \\
\hline 5324 & 3.19 down PLAG1 & pleiomorphic adenoma gene 1 \\
\hline 56937 & 2.59 down PMEPA1 & prostate androgen induced (TMEPAI) \\
\hline 64089 & 2.90 down SNX16 & sorting nexin 16 (SNX16) \\
\hline 10252 & 3.62 down SPRY1 & Sprouty 1 \\
\hline 8115 & 4.81 down TCL1A & T-cell leukemia/lymphoma $1 \mathrm{~A}$ \\
\hline 27075 & 2.64 down TSPAN13 & tetraspanin NET- 6 protein (NET-6) \\
\hline \multicolumn{3}{|c|}{ Immune-Related } \\
\hline 55024 & 3.66 down BANK1 & B-cell scaffold protein with ankyrin repeats 1 \\
\hline 973 & 3.22 down CD79A & B-cell antigen receptor (MB-1) \\
\hline 3563 & 2.46 up IL3RA & interleukin 3 receptor, alpha \\
\hline 3576 & 8.03 down IL8 & interleukin 8 (IL8) \\
\hline 115350 & 4.24 down FCRL1 & Fc receptor like 1 (CD307a) \\
\hline 79368 & 3.24 down FCRL2 & $\mathrm{SH} 2$ domain-containing phosphatase anchor \\
\hline 3120 & 3.68 up HLA-DQB2 & BCG responsive, SLE associated \\
\hline 10561 & 2.82 down IFI44 & interferon-induced, hep C-associated protein \\
\hline 64108 & 4.07 down RTP4 & interferon responsive protein (IFRG28) \\
\hline 26228 & 3.39 down STAP1 & signal transducing adapter 1 (BRDG1) \\
\hline 340206 & 3.65 up TREML3 & triggering receptor expressed on myeloid cel \\
\hline
\end{tabular}

\section{Electrolyte Transporters}

$\begin{array}{rr}1183 & 2.29 \text { down CLCN4 } \\ 131096 & 4.70 \text { down KCNH8 } \\ 56749 & 2.05 \text { down KCNQ5 } \\ 6328 & 4.99 \text { down SCN3A }\end{array}$

Signaling/Detoxifying Enzymes

$2052 \quad 2.21$ up EPHX1

$5190 \quad 2.02$ up PEX6

$5730 \quad 2.12$ up PTGDS

Drug transport

$5244 \quad 2.14$ down $\mathrm{ABCB} 4$

52432.14 down ABCB1 chloride channel 4

potassium voltage-gated channel, $\mathrm{H} 8$

voltage-gated potassium channel

Nav1.3 sodium channel

epoxide hydrolase 1 , microsomal peroxisomal biogenesis factor 6

prostaglandin D2 synthase

MDR2/3, multidrug resistance protein 3

P-glycoprotein (mdr1) mRNA

\section{Gene Expression Profiling of Whole Blood RNA}

Expression profiling of blood indicates that women who developed a low EF after chemotherapy had 260 transcripts that differed $>2$-fold, with a t-test probability of $p<0.05$, compared to women receiving chemo who maintained normal EFs (A vs. B). Most of these transcripts (201) were not shared by women who developed low EFs independent of chemotherapy (C vs. D). As shown in Figure 2, the 201 transcripts that differentiate the chemo-induced low EF women (A) from women similarly treated, but retaining normal EF (B), tend to be more down-regulated (blue) than up-regulated (red). However, these differentially expressed transcripts are not similarly regulated in women with low EFs who never received chemo (C). However, 59 transcripts were identified that were differentially expressed in both chemo-dependent (A vs. B) and chemo-independent low EFs (C vs. D), suggesting these transcripts report some general cause or effect of low EFs (Additional file 2: Supplementary Tables 1 and 2). Selected transcripts from the 201 gene list, organized by putative function, are shown in Table 2 , and discussed below.

Functional annotation: Analysis of the DEG list in DAVID and IPA helped to identify relationships between the transcripts. In the 'biological processes' category, 18 terms were statistically overrepresented, with transcripts mainly categorized into cellular activation, cellular proliferation, immune response, differentiation, signal transduction, and drug response/transport. With additional manual annotation, several general pathways with potential relevance to heart failure emerged.

Apoptosis: Among the DEGs unique to chemo-induced low EFs, there were notable changes in apoptosis-related transcripts. There was decreased 
expression of BIRC3, which is a member of the inhibitor of apoptosis protein family (cIAP2, IAP-C, $\mathrm{MIHC}$ ), which are ubiquitin ligases that target the degradation of the TNF receptor interacting protein (RIP)[17]. Conversely, there was decreased expression of the proapoptotic caspase 8 associated protein 2 (CASP8AP2, FLASH, Flice-associated huge protein), which facilitates caspase 8 activation by TNF receptor or fas [18]. Interestingly, CASP8AP2 has been identified as mediating the cardiac ischemic preconditioning effect of mir210 [19]. PLAG1, decreased more than 3 -fold, is a proto-oncogene transcription factor that regulates numerous apoptotic signals [20].

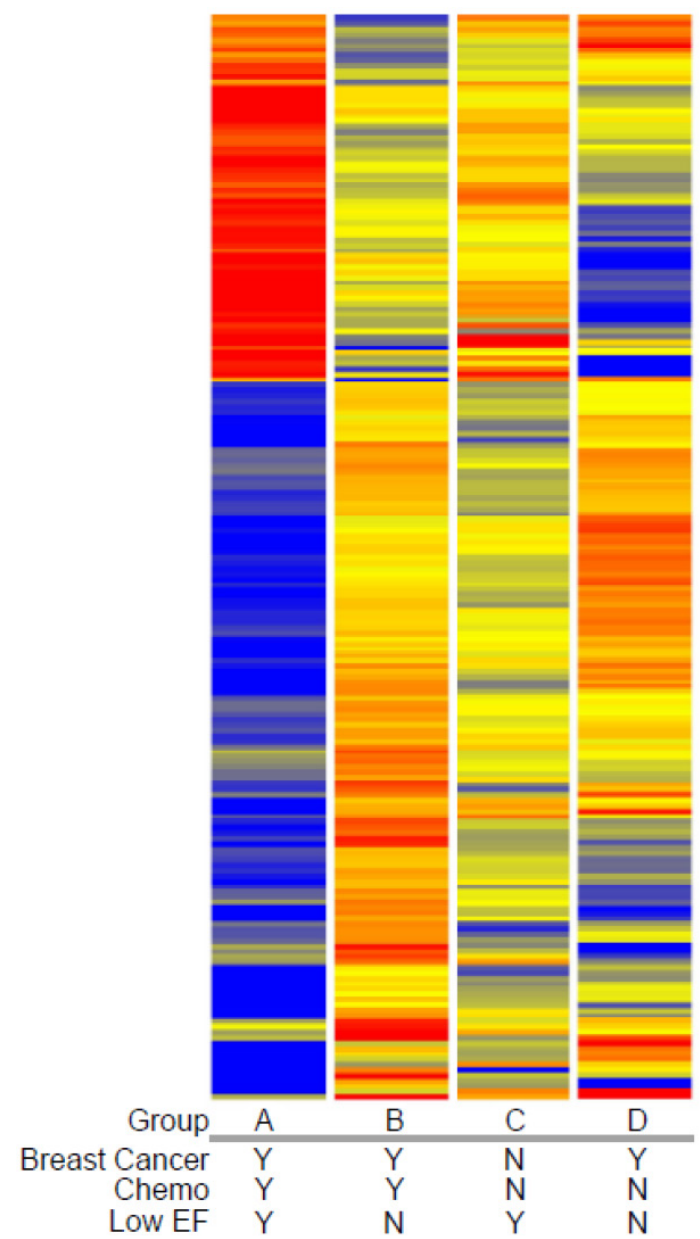

Fig 2. Clustered heatmap of transcripts associated with chemotherapy-induced heart failure. Transcript profiles of blood from women with breast cancer who did (Group A) or did not (Group B) develop low EFs were compared to identify differentially-expressed genes (DEGs) by a combined fold-change $(>1.5$ fold $)$ and t-test $(p<0.05)$ filtering. To exclude transcripts that were associated with heart failure in women not receiving doxorubicin, women with breast cancer and normal cardiac performance were sampled prior to chemotherapy (Group D) and compared to women with low EFs but who never received chemotherapy (Group C). The resulting 201 transcripts were clustered by their expression pattern across the 4 groups with each row indicating one transcript. Red indicates increased expression, blue indicates decreased expression, with yellow indicating little or no change.
Notably, women with chemo-induced low EFs had a 4.8-fold decrease in the oncogene T-cell leukemia/lymphoma 1A (TCL1A). TCL1A is expressed in both cardiac and skeletal muscle, and is a known co-activator for $\mathrm{AKT}$, one of the major pro-survival factors for cardiomyocytes. Additionally, genome-wide SNP analysis has previously identified TCL1A SNPs strongly associated with musculoskeletal adverse events in women receiving aromatase inhibitors (AI) [21]. The variant allele at rs11849538 is quite common (22-34\% heterozygous, 6\% homozygous), and creates a new estrogen receptor-binding site [21].

Immune-related: Considering that whole blood was profiled, it is reasonable that the majority of differentially-expressed transcripts fall into immune-related categories, particularly T-cell and B-cell activation and proliferation. However, recent data has suggested that hyper-activated innate immune responses could contribute to the progression of $\mathrm{CHF}$ (reviewed in [22]). Notably, there was a 2.5-fold increase in the interleukin-3 receptor (IL3R), and an 8 -fold decrease in IL-8, a change twice as large as any other transcript. Expression levels in general, however, did not support a general activation of innate immunity in circulating cells, with consistent down-regulation of B-cell activation markers (BANK1, CD79A), Fc-like receptors (FCRL1, FCRL2), and two interferon-responsive transcripts (IFI44, RTP4/IFRG28). However, elevated expression of HLA-DQB2 could suggest some type of autoimmune reaction triggered by chemotherapy, because SNPs in DQB2 have been associated with rheumatoid arthritis [23] and lupus [24].

Electrolyte transporters: Another potential explanation for the increased cardiotoxicity in some women would be if there were inherited or acquired defects in membrane transporters for electrolytes, which could create performance deficits that would then be exacerbated by chemotherapy, leading to cell death. There were noticeable changes in four electrolyte channels: chloride channel 4 (CLCN4), Nav1.3 sodium channel (SCN3A), and two voltage-gated potassium channels (KCNH8, KCNQ5).

Signaling/Detoxifying Enzymes: Interestingly, the microsomal epoxide hydrolase (EPHX1), which is a known xenobiotic metabolizing enzyme for by-products of doxorubicin, was elevated 2.2-fold in the chemo-CHF group. Prior genotyping studies observed that EPHX1 polymorphisms were associated with both breast cancer risk and overall survival after therapy [25]. PEX6 was elevated 2-fold and plays a key role in the assembly of the peroxisome. Defects in PEX6 lead to elevated free radicals and cellular ne- 
crosis [26], suggesting that elevated PEX6 may be a compensatory response to increased free radical levels. Prostaglandin D2 synthase (PTGDS) mRNA was elevated 2.1-fold in the chemo-CHF cases, and the PTGDS protein has recently been reported as a circulating biomarker in coronary artery disease [27; 28] and PTGDS mRNA is elevated in epicardial adipose tissue of patients with coronary disease [29].

Drug Transport: Among the most straightforward explanations for increased cardiotoxicity would be elevated cardiac levels of doxorubicin, possibly secondary to decreased multidrug resistance proteins in the cardiac tissue. Thus, it is interesting that both multidrug resistance protein 1 (MDR1, p-glycoprotein, ABCB1) and MDR2/3 (ABCB4) mRNAs in blood were decreased more than 2-fold in the chemo patients that developed low EFs. MDR1 is a well-established efflux pump for doxorubicin [30]. Elevated levels of MDR1 in tumor cells [31], and promoter SNPs that increase MDR1 expression, are commonly part of the chemotherapy-resistant phenotype [32]. Acute cardiotoxicity of doxorubicin has previously been linked to G671V variants of MDR1 in case-control studies [33]. While it is well known that doxorubicin is a substrate for MDR1, recent in vitro analysis also suggests that this coding variant decreases the efflux of free radical adducts from cells [34]. Thus, if cardiac levels of MDR1 and MDR2/4 were reduced in parallel to the blood levels, the cells may experience both elevated doxorubicin levels and elevated retention of the induced free radicals.

\section{Free Radical Levels}

To determine whether doxorubicin caused elevations in free radical formation, a substudy was conducted on patients in which plasma samples were obtained pre-chemo, and 24 hours after at least five chemo cycles. As shown in Figure 3, plasma TBARS increased up to 2.5-fold after chemotherapy in some patients, with average levels elevated $64 \%$ in the four patients evaluated (ANOVA $\mathrm{p} \leq 0.01$ ). Despite elevated free radicals, none of these patients developed cardiomyopathy or low EF within the study period. This confirms prior data demonstrating elevated plasma TBARS post-chemo [35], and extends it to demonstrate that the elevations are sustained over five chemo cycles.

\section{Genotyping Analysis}

The almost 5-fold reduction in TCL1A mRNA levels in the chemo-CHF group suggested that it might contribute to increased cardiac apoptosis. Genome-wide SNP analysis has previously identified TCL1A SNPs strongly associated with musculoskele- tal adverse events in women receiving aromatase inhibitors (AI) [21]. The variant allele (G) at rs11849538 is quite common (HapMap variant allele frequency of $8-43 \%)$, and creates an estrogen receptor-binding site. However, genotyping for this SNP in 18 patients, including all of the women with chemo-induced low EFs (Group A), did not detect differential variant allele frequencies, with essentially all groups near $40 \%$ (not shown).

\section{Effect of MDRI inhibition on response to dox- orubicin in vitro}

To determine whether the reduced MDR1 could have a functional effect on cardiomyocyte survival, H9C2 rat embryonic cardiomyocytes were pretreated with verapamil $(0-100 \mu \mathrm{M})$, a known MDR1 inhibitor with an effective $\mathrm{IC}_{50}$ of $120 \mu \mathrm{M}$ [36], 2 hours prior to low doses of doxorubicin $(0-50 \mu \mathrm{M})$. Pretreatment with verapamil significantly enhanced the toxicity of doxorubicin (ANOVA $\mathrm{p}<0.001$ for both main effects and interaction), which was significant in pairwise testing at the combined doses of $50-100 \mu \mathrm{m}$ verapamil and $10-50 \mu \mathrm{M}$ doxorubicin in $\mathrm{H} 9 \mathrm{C} 2$ cardiomyocytes (Figure 4). This confirms prior studies indicating that inhibition of MDR1 activity by verapamil increases the toxic effect of doxorubicin in cardiomyocytes [37-40].

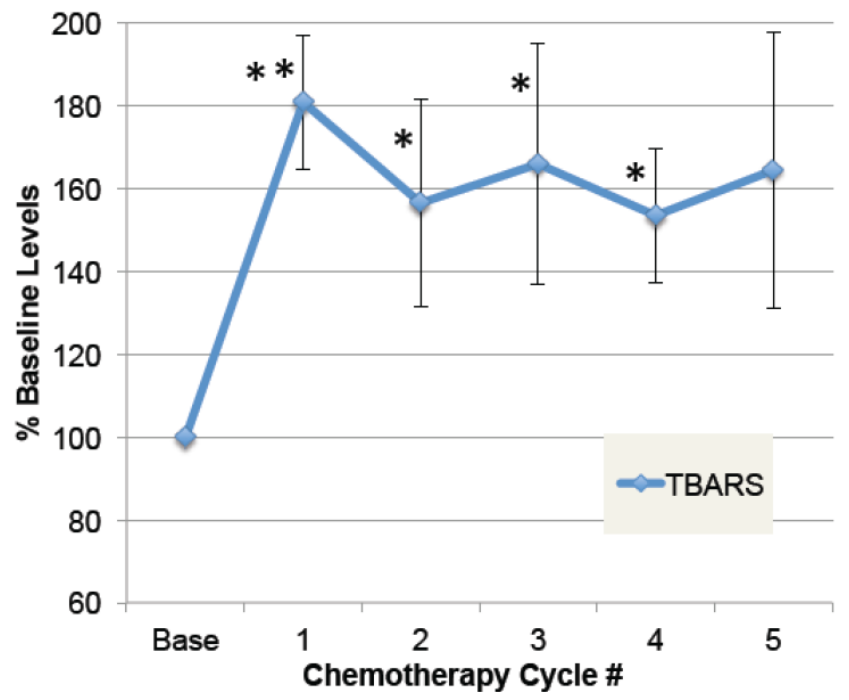

Fig 3. Plasma levels of free radical modifications to protein. A small group of patients were followed prior to doxorubicin-based chemotherapy and then after multiple cycles of chemotherapy. Plasma samples, pre-chemo, and 24 hours after each chemo cycle, were specially preserved, stored at $-80^{\circ} \mathrm{C}$, and then analyzed for products of oxidative modification. Thiobarbituric-acid reactive substances (TBARS) in plasma are shown for pre-chemo (Base) and 5 subsequent chemo cycles (error bars $=$ s.e.m.). The main effect of chemotherapy on TBARS, expressed as a percent of baseline values, was evaluated by ANOVA $(p=0.01)$, and asterisks indicate where post-hoc t-tests were significant compared to pre-chemo baseline $(* * p \leq 0.01, * p \leq 0.05, n=4)$. 


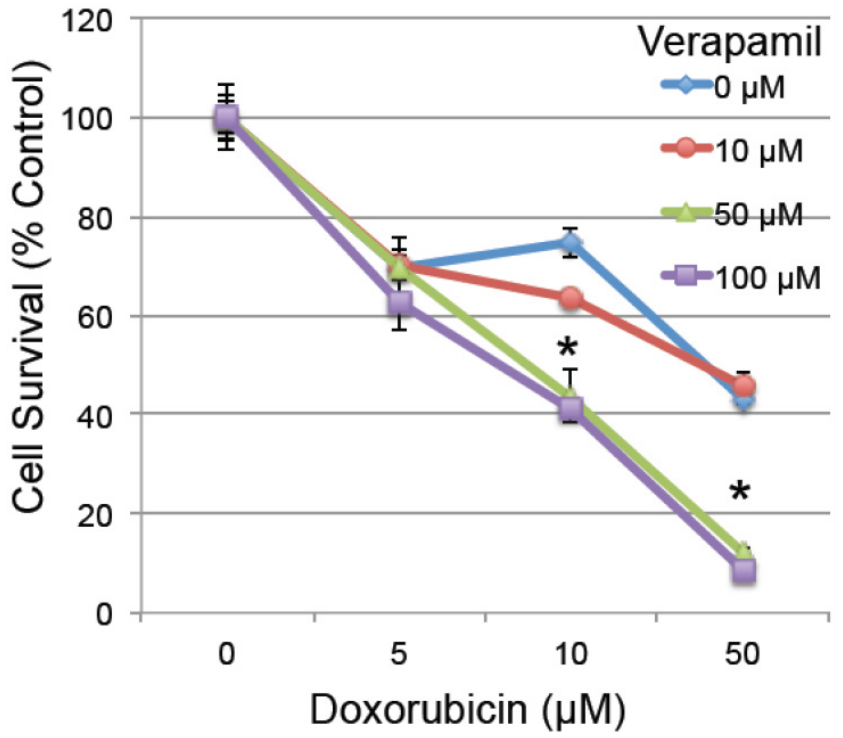

Fig 4. The effect of MDRI inhibition on doxorubicin-induced cell death. Rat $\mathrm{H} 9 \mathrm{C} 2$ cardiomyocytes were pretreated with the MDRI inhibitor verapamil for 2 hours at the specified doses $(0-100 \mu \mathrm{M})$. Cells were then treated with increasing doses of doxorubicin $(0-50 \mu \mathrm{M})$ and cell survival was determined 20 hours later by measuring their metabolic reduction of MTT, with the product measured by O.D. at $570 \mathrm{~nm}$ $(n=3$ /group, error bars $=$ s.e.m.). Asterisk indicates a significant change between verapamil treated and untreated $(p \leq 0.05)$.

\section{Discussion}

The general mechanisms of chemo-induced heart failure have been extensively studied and two major etiologies have emerged: 1) direct damage to the cardiomyocyte's DNA, triggering apoptosis, and 2) induction and accumulation of cellular free radicals causing toxicity and cardiomyocyte death. However, the particular inter-individual variations that affect the susceptibility to chemo-induced CHF are poorly understood. Pretreatment variables, such as age and left ventricular EF have been identified as predictors of the adverse cardiac response to epirubicin, suggesting that a pre-existing, but compensated cardiac performance deficit may increase the susceptibility to chemotherapy-induced damage [16].

Several lines of evidence suggest that the survival/apoptosis pathways are a major determinant of the cardiac outcome after doxorubicin. In rat neonatal cardiomyocytes, overexpression of the apoptosis regulator ARC inhibits doxorubicin-induced death, while siRNA to ARC increases apoptosis induced by doxorubicin [41], suggesting that doxorubicin induces death via caspase-dependent mechanisms. In a rat model, intracoronary AKT1 gene delivery mitigates doxorubicin-induced reductions in fractional shortening, and improves LV function at five weeks post-treatment [42]. The apparent increase in cardio- toxicity associated with obesity led to pre-clinical studies in which adiponectin was shown to reduce cardiac dysfunction after doxorubicin by its ability to stimulate AKT activity [43]. Likewise, microarray analysis of cardiac tissue in mice developing LV dysfunction after doxorubicin treatment showed changes in transcripts associated with hypertrophy (STARS), ATP levels (SNF1-kinase), and apoptosis, (AXUD1, a downstream target of AXIN1) [44]. Thus, the cardiac levels of TCL1A may be a critical determinant of AKT activity in the cardiomyocytes, and thus a key factor in susceptibility to doxorubicin cardiotoxicity. It is interesting to speculate that the reduced blood levels of TCL1A might be related to the known effect of Epstein-Barr Virus (EBV) latent membrane protein1 (LMP1), which suppresses TCL1A levels via miRNA 29-b [45].

The second major pathway of cardiac damage, free radical injury, is influenced by changes in antioxidant systems, and variations in the efflux pumps for doxorubicin and the free radicals that it creates. Polymorphisms in ABCB1/MDR1, such as C3435T, are known to affect mRNA levels in breast cancer specimens [46] and may be related to therapeutic outcome from anthracyclines [32; 47], possibly by affecting mRNA stability [48]. However, the relationship between MDR1 polymorphisms and mRNA expression or drug transport remains complicated [49]. For instance, cardiac levels of MDR1 are affected by an exon 21 polymorphism, but not by the C3435T SNP [50]. Transgenic mice overexpressing MDR1 selectively in the myocardium are protected from damage inflicted by doxorubicin [51]. Further, knockout of heat shock factor 1 (HSF1) increased the expression of MDR1 in the heart, increased efflux of doxorubicin, and reduced mortality [40]. MDR1 levels could be modulated by AKT activity because signaling through phosphoinositide 3-kinase (PI3K) and AKT can increase MDR1 expression [52], which is consistent with the reduction in both TCL1A and MDR1 in the present studies. Recent data in experimental models has suggested that one important factor might be the impact of the chemotherapy on the cardiac stem/progenitor cells. When syngeneic cardiac progenitors were reintroduced post-chemo in mice, cardiac performance was restored [53]. It is worth noting that MDR1 protein is considered one of the key markers of cardiac stem cells [54].

The present studies have several limitations that deserve recognition. First, this is a relatively small sample size, with only five women who have chemo-induced low EFs: a consequence of the fact that oncologists have been aware of this complication and reduce the chemo dosages to render this a rela- 
tively rare side effect. Second, of necessity, the studies employed a surrogate marker for expression profiling, peripheral blood, which may or may not reflect the expression profile in the heart. Based on the present studies, it would be important to investigate whether cardiac MDR1 levels, or MDR-like efflux activity, are predictive of cardiomyopathy after chemotherapy. Third, because of the low incidence of chemo-induced $\mathrm{HF}$, it was impractical to sample patients prior to the initiation of chemo, and then follow them to determine differences between women who did, of did not develop low EFs. Rather, it was more practical to sample patients with low EFs after chemotherapy, and compare them with patients with normal EF. The general effect of low EF on expression profiles was then determined, and subtracted, by analyzing non-chemo patients with low EF.

\section{TCL1A/MDR1 Hypothesis:}

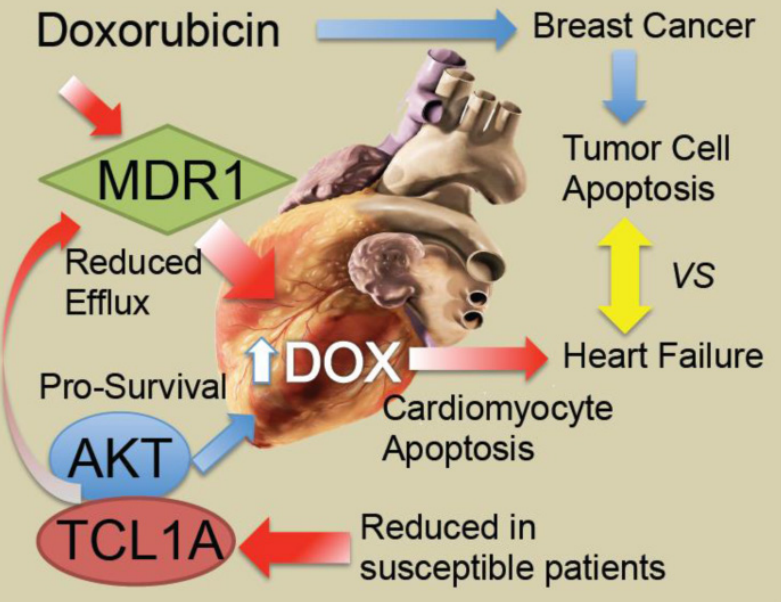

Fig 5. Schematic model of TCLIA and MDRI effects on the cardiac response to chemotherapy. Doxorubicin and related anthracyclines intercalate on DNA and induce DNA damage, as well as inducing free radical production, combining to preferentially induce the death of rapidly dividing tumor cells. However, cardiomyocytes may also undergo apoptosis, leading to decreased cardiac performance, and additional stress on the remaining cardiomyocytes. The present studies suggest that reduced cardiac expression of TCLIA leads to a reduced survival and protective effect of AKTI, making the cells more sensitive to apoptosis. Further, reduced cardiac expression of TCLIA/AKT could lead to reduced $A B C B I / M D R I$, and $A B C B 4$, drug efflux pumps for doxorubicin, which would be expected to increase the intracellular levels of doxorubicin, as well as reducing the ability of the cardiomyocytes to export the free radicals that doxorubicin induces.

\section{Conclusions}

Doxorubicin-induced cardiotoxicity remains a devastating complication of a potentially life-saving drug. As summarized in Figure 5, it is proposed that chemo-induced cardiomyopathy may be due to a genetic predisposition or progressively acquired tendency to reduced TCL1A levels, reducing AKT activity and thereby causing increased apoptotic sensitivity to doxorubicin. Furthermore, it is possible that coincidental or AKT-related changes in MDR1 levels in the myocardium decrease the efflux of doxorubicin, thereby causing its accumulation, along with toxic free radicals in the cardiomyocytes. Future studies will be needed to determine whether screening for blood levels of TCL1A and MDR1 mRNA are helpful to identify women at greatest risk of chemo-induced heart failure.

\section{Abbreviations}

$\mathrm{CHF}$, congestive heart failure; DEG, differential expressed genes; EF, ejection fraction; EGF, epidermal growth factor; ESP, end-stage pressure; ESV, end-systolic volume; FBS, fetal bovine serum; HSF1, heat shock factor 1 ; LV, left ventricular; MDR1, multidrug resistance protein 1; mRNA, messenger RNA; PI3K, phosphoinositide 3-kinase; siRNA, small interfering RNA; SNP, single nucleotide polymorphisms; SW, stroke work; TBARS, thiobarbituric acid reactive substances; TCL1A, T-cell leukemia/lymphoma 1A.

\section{Supplementary Material}

Additional File 1:

Supplementary Figure 1 presents an echocardiography from typical patients with, and without, chemo-induced cardiomyopathy. http://www.ijbs.com/v09p0350s1.pdf Additional File 2:

Supplementary Table 1 lists all 260 transcripts differentially expressed between Group A and Group B, with a sub-listing of the 59 transcripts differentially expressed in both chemo-dependent (A vs. B) and chemo-independent low EF (C vs. D). Supplementary Table 2 lists all 1531 transcripts differentially expressed between Group C and Group D. All original Affymetrix .cel files, as well as the summarized RMA raw data, and per gene normalized data have been deposited at NCBI Gene Expression Omnibus (GEO) under the Accession \# GSE40447.

http://www.ijbs.com/v09p0350s2.xlsx

\section{Acknowledgements}

This work was supported by The GWU Heart and Vascular Institute. The authors are grateful to all of the research volunteers who selflessly participated as subjects in this study despite their serious medical situations. The heart graphic incorporated into Figure 5 is courtesy of Patrick J. Lynch, medical illustrator; C. 
Carl Jaffe, MD, cardiologist, Yale University, via WikiCommons.

\section{Competing interests}

TM holds an equity interest in Cellgenex, Inc. a privately-held genomics company developing RNA-based diagnostics tests in the cardiovascular area. However, the present studies are not supported by Cellgenex, thus there are no competing interests that would affect the results of these studies. The other authors declare no competing interests.

\section{References}

1. Singal PK, Iliskovic N. Doxorubicin-induced cardiomyopathy. New Engl J Med. 1998; 339(13): 900-905.

2. Shan K, Lincoff AM, Young JB. Anthracycline-induced cardiotoxicity. Ann Int Med. 1996; 125(1): 47-58.

3. Chien KR. Herceptin and the heart--a molecular modifier of cardiac failure. New Engl J of Med. 2006; 354(8): 789-790.

4. Slamon DJ, Leyland-Jones B, Shak S, Fuchs H, Paton V, Bajamonde A, Fleming T, Eiermann W, Wolter J, Pegram M et al. Use of chemotherapy plus a monoclonal antibody against HER2 for metastatic breast cancer that overexpresses HER2. New Engl J Med. 2001; 344(11): 783-792.

5. Kobayashi S, Ito A, Okuzaki D, Onda H, Yabuta N, Nagamori I, Suzuki $\mathrm{K}$, Hashimoto $\mathrm{H}$, Nojima $\mathrm{H}$. Expression profiling of PBMC-based diagnostic gene markers isolated from vasculitis patients. DNA Res. 2008; 15(4): 253-265.

6. Arasappan D, Tong W, Mummaneni P, Fang H, Amur S. Meta-analysis of microarray data using a pathway-based approach identifies a 37-gene expression signature for systemic lupus erythematosus in human peripheral blood mononuclear cells. BMC Med. 2011; 9: 65.

7. Healy AM, Pickard MD, Pradhan AD, Wang Y, Chen Z, Croce K, Sakuma M, Shi C, Zago AC, Garasic J et al. Platelet expression profiling and clinical validation of myeloid-related protein-14 as a novel determinant of cardiovascular events. Circulation. 2006; 113(19): 2278-2284.

8. Rosenberg S, Elashoff MR, Beineke P, Daniels SE, Wingrove JA, Tingley WG, Sager PT, Sehnert AJ, Yau M, Kraus WE et al. Multicenter validation of the diagnostic accuracy of a blood-based gene expression test for assessing obstructive coronary artery disease in nondiabetic patients. Ann Intern Med. 2010; 153(7): 425-434.

9. Liew CC. Expressed genome molecular signatures of heart failure. Clin Chem Lab Med. 2005; 43(5): 462-469.

10. Nagueh SF, Middleton KJ, Kopelen HA, Zoghbi WA, Quinones MA. Doppler tissue imaging: a noninvasive technique for evaluation of left ventricular relaxation and estimation of filling pressures. J Am Coll Cardiol. 1997; 30(6): 1527-1533.

11. Yu CM, Gorcsan J, 3rd, Bleeker GB, Zhang Q Schalij MJ, Suffoletto MS, Fung JW, Schwartzman D, Chan YS, Tanabe M et al. Usefulness of tissue Doppler velocity and strain dyssynchrony for predicting left ventricular reverse remodeling response after cardiac resynchronization therapy. Am J Cardiol. 2007; 100(8): 1263-1270.

12. Mak IT, Weglicki WB. Antioxidant activity of calcium channel blocking drugs. Methods Enzymol. 1994; 234: 620-630.

13. Huang da W, Sherman BT, Lempicki RA. Systematic and integrative analysis of large gene lists using DAVID bioinformatics resources. Nat Protoc. 2009; 4(1): 44-57.

14. Andolfatto S, Namour F, Garnier AL, Chabot F, Gueant JL, Aimone-Gastin I. Genomic DNA extraction from small amounts of serum to be used for alpha1-antitrypsin genotype analysis. Eur Respir J. 2003; 21(2): 215-219.

15. Heo DS, Park JG, Hata K, Day R, Herberman RB, Whiteside TL. Evaluation of tetrazolium-based semiautomatic colorimetric assay for measurement of human antitumor cytotoxicity. Cancer Res. 1990; 50: 3681-3690.

16. Schaadt B, Kelbaek $H$. Age and left ventricular ejection fraction identify patients with advanced breast cancer at high risk for development of epirubicin-induced heart failure. J Nucl Cardiol. 1997; 4(6): 494-501.

17. Park SM, Yoon JB, Lee TH. Receptor interacting protein is ubiquitinated by cellular inhibitor of apoptosis proteins (c-IAP1 and c-IAP2) in vitro. FEBS Lett. 2004; 566(1-3): 151-156.
18. Imai $Y$, Kimura T, Murakami A, Yajima N, Sakamaki K, Yonehara S. The CED-4-homologous protein FLASH is involved in Fas-mediated activation of caspase-8 during apoptosis. Nature $1999 ; 398(6730)$ : 777-785.

19. Kim HW, Haider HK, Jiang S, Ashraf M. Ischemic preconditioning augments survival of stem cells via miR-210 expression by targeting caspase-8-associated protein 2. J Biol Chem. 2009; 284(48): 33161-33168.

20. Voz ML, Mathys J, Hensen K, Pendeville H, Van Valckenborgh I, Van Huffel C, Chavez M, Van Damme B, De Moor B, Moreau Y et al. Microarray screening for target genes of the proto-oncogene PLAG1. Oncogene 2004; 23(1): 179-191.

21. Ingle JN, Schaid DJ, Goss PE, Liu M, Mushiroda T, Chapman JA, Kubo M, Jenkins GD, Batzler A, Shepherd L et al. Genome-wide associations and functional genomic studies of musculoskeletal adverse events in women receiving aromatase inhibitors. J Clin Oncol. 2010; 28(31): 4674-4682.

22. Shi Y, Moon M, Dawood S, McManus B, Liu PP. Mechanisms and management of doxorubicin cardiotoxicity. Herz 2011; 36(4): 296-305.

23. Kochi Y, Yamada R, Kobayashi K, Takahashi A, Suzuki A, Sekine A, Mabuchi A, Akiyama F, Tsunoda T, Nakamura Y et al. Analysis of single-nucleotide polymorphisms in Japanese rheumatoid arthritis patients shows additional susceptibility markers besides the classic shared epitope susceptibility sequences. Arthritis Rheum 2004; 50(1): 63-71.

24. Barcellos LF, May SL, Ramsay PP, Quach HL, Lane JA, Nititham J, Noble JA, Taylor KE, Quach DL, Chung SA et al. High-density SNP screening of the major histocompatibility complex in systemic lupus erythematosus demonstrates strong evidence for independent susceptibility regions. PLoS Genet 2009; 5(10): e1000696.

25. Khedhaier A, Hassen E, Bouaouina N, Gabbouj S, Ahmed SB, Chouchane L. Implication of Xenobiotic Metabolizing Enzyme gene (CYP2E1, CYP2C19, CYP2D6, mEH and NAT2) polymorphisms in breast carcinoma. BMC Cancer 2008; 8: 109.

26. Jungwirth $H$, Ring J, Mayer $T$, Schauer A, Buttner S, Eisenberg $T$, Carmona-Gutierrez D, Kuchler K, Madeo F. Loss of peroxisome function triggers necrosis. FEBS Lett 2008; 582(19): 2882-2886.

27. Inoue $\mathrm{T}$, Eguchi $\mathrm{Y}$, Matsumoto $\mathrm{T}$, Kijima $\mathrm{Y}$, Kato Y, Ozaki Y, Waseda K, Oda H, Seiki K, Node K et al. Lipocalin-type prostaglandin D synthase is a powerful biomarker for severity of stable coronary artery disease. Atherosclerosis 2008; 201(2): 385-391.

28. Miwa Y, Oda H, Shiina Y, Shikata K, Tsushima M, Nakano S, Maruyama $\mathrm{T}$, Kyotani S, Eguchi N, Urade $\mathrm{Y}$ et al. Association of serum lipocalin-type prostaglandin D synthase levels with subclinical atherosclerosis in untreated asymptomatic subjects. Hypertens Res. 2008; 31(10): 1931-1939.

29. Guauque-Olarte S, Gaudreault N, Piche ME, Fournier D, Mauriege P, Mathieu P, Bosse Y. The transcriptome of human epicardial, mediastinal and subcutaneous adipose tissues in men with coronary artery disease. PLoS One 2011; 6(5): e19908.

30. Ueda K, Cardarelli C, Gottesman MM, Pastan I. Expression of a full-length cDNA for the human "MDR1" gene confers resistance to colchicine, doxorubicin, and vinblastine. Proc Natl Acad Sci USA. 1987;84(9): 3004-3008.

31. Krech T, Scheuerer E, Geffers R, Kreipe H, Lehmann U, Christgen M. ABCB1/MDR1 contributes to the anticancer drug-resistant phenotype of IPH-926 human lobular breast cancer cells. Cancer Lett. 2012; 315(2): 153-160.

32. George J, Dharanipragada K, Krishnamachari S, Chandrasekaran A, Sam $\mathrm{SS}$, Sunder E. A single-nucleotide polymorphism in the MDR1 gene as a predictor of response to neoadjuvant chemotherapy in breast cancer. Clinical Breast Cancer 2009; 9(3): 161-165.

33. Wojnowski L, Kulle B, Schirmer M, Schluter G, Schmidt A, Rosenberger A, Vonhof S, Bickeboller H, Toliat MR, Suk EK et al. NAD $(\mathrm{P}) \mathrm{H}$ oxidase and multidrug resistance protein genetic polymorphisms are associated with doxorubicin-induced cardiotoxicity. Circulation 2005; 112(24): 3754-3762.

34. Jungsuwadee $\mathrm{P}$, Zhao T, Stolarczyk EI, Paumi CM, Butterfield DA, St Clair DK, Vore M. The G671V variant of MRP1/ABCC1 links doxorubicin-induced acute cardiac toxicity to disposition of the glutathione conjugate of 4-hydroxy-2-trans-nonenal. Pharmacogenet Genomics 2012; 22(4): 273-284.

35. Panis C, Herrera AC, Victorino VI, Campos FC, Freitas LF, De Rossi T, Colado Simao AN, Cecchini AL, Cecchini $R$. Oxidative stress and hematological profiles of advanced breast cancer patients subjected to paclitaxel or doxorubicin chemotherapy. Breast Cancer Res Treat 2012. 133(1): 89-97. 
36. Kars MD, Iseri OD, Gunduz U, Molnar J. Reversal of multidrug resistance by synthetic and natural compounds in drug-resistant MCF-7 cell lines. Chemotherapy 2008; 54(3): 194-200.

37. Gruber A, Larsson R, Nygren P, Bjorkholm M, Peterson C. A non-P-glycoprotein-mediated mechanism of vincristine transport which is affected by resistance modifiers and present in chemosensitive cells. Leukemia 1994; 8(6): 985-989.

38. Estevez MD, Wolf A, Schramm U. Effect of PSC 833, verapamil and amiodarone on adriamycin toxicity in cultured rat cardiomyocytes. Toxicol In Vitro 2000; 14(1): 17-23.

39. Farokhpour M, Karbalaie K, Tanhaei S, Nematollahi M, Etebari M, Sadeghi HM, Nasr-Esfahani MH, Baharvand H. Embryonic stem cell-derived cardiomyocytes as a model system to study cardioprotective effects of dexamethasone in doxorubicin cardiotoxicity. Toxicol In Vitro 2009; 23(7): 1422-1428.

40. Krishnamurthy K, Vedam K, Kanagasabai R, Druhan LJ, Ilangovan G. Heat shock factor-1 knockout induces multidrug resistance gene, MDR1b, and enhances P-glycoprotein (ABCB1)-based drug extrusion in the heart. Proc Natl Acad Sci U S A. 2012; 109(23):9023-8.

41. An J, Li P, Li J, Dietz R, Donath S. ARC is a critical cardiomyocyte survival switch in doxorubicin cardiotoxicity. J Mol Med (Berl) 2009; 87(4): 401-410.

42. Taniyama Y, Walsh K. Elevated myocardial Akt signaling ameliorates doxorubicin-induced congestive heart failure and promotes heart growth. J Mol Cell Cardiol 2002; 34(10): 1241-1247.

43. Maruyama S, Shibata R, Ohashi K, Ohashi T, Daida H, Walsh K, Murohara T, Ouchi N. Adiponectin ameliorates doxorubicin-induced cardiotoxicity through Akt protein-dependent mechanism. J Biol Chem. 2011; 286(37): 32790-32800.

44. Yi X, Bekeredjian R, DeFilippis NJ, Siddiquee Z, Fernandez E, Shohet RV. Transcriptional analysis of doxorubicin-induced cardiotoxicity. Am J Physiol Heart Circ Physiol 2006; 290(3): H1098-1102.

45. Anastasiadou E, Boccellato F, Vincenti S, Rosato P, Bozzoni I, Frati L, Faggioni A, Presutti C, Trivedi P. Epstein-Barr virus encoded LMP1 downregulates TCL1 oncogene through miR-29b. Oncogene 2010; 29(9): 1316-1328.

46. Taheri M, Mahjoubi F, Omranipour R. Effect of MDR1 polymorphism on multidrug resistance expression in breast cancer patients. Genet Mol Res. 2010; 9(1): 34-40.

47. Cizmarikova M, Wagnerova M, Schonova L, Habalova V, Kohut A, Linkova A, Sarissky M, Mojzis J, Mirossay L, Mirossay A. MDR1 (C3435T) polymorphism: relation to the risk of breast cancer and therapeutic outcome. Pharmacogenomics Journal 2010; 10(1): 62-69.

48. Wang D, Johnson AD, Papp AC, Kroetz DL, Sadee W. Multidrug resistance polypeptide 1 (MDR1, ABCB1) variant 3435C $>\mathrm{T}$ affects mRNA stability. Pharmacogenet Genomics 2005; 15(10): 693-704.

49. Sakaeda T. MDR1 genotype-related pharmacokinetics: fact or fiction? Drug Metab Pharmacokinet 2005; 20(6): 391-414.

50. Meissner K, Jedlitschky G, Meyer zu Schwabedissen H, Dazert P, Eckel L, Vogelgesang S, Warzok RW, Bohm M, Lehmann C, Wendt M et al. Modulation of multidrug resistance P-glycoprotein 1 (ABCB1) expression in human heart by hereditary polymorphisms. Pharmacogenetics 2004; 14(6): 381-385.

51. Dell'Acqua G, Polishchuck R, Fallon JT, Gordon JW. Cardiac resistance to adriamycin in transgenic mice expressing a rat alpha-cardiac myosin heavy chain/human multiple drug resistance 1 fusion gene. Hum Gene Ther 1999; 10(8): 1269-1279.

52. Kuo MT, Liu Z, Wei Y, Lin-Lee YC, Tatebe S, Mills GB, Unate H. Induction of human MDR1 gene expression by 2-acetylaminofluorene is mediated by effectors of the phosphoinositide 3-kinase pathway that activate NF-kappaB signaling. Oncogene 2002; 21(13): 1945-1954.

53. De Angelis A, Piegari E, Cappetta D, Marino L, Filippelli A, Berrino L, Ferreira-Martins J, Zheng H, Hosoda T, Rota M et al. Anthracycline cardiomyopathy is mediated by depletion of the cardiac stem cell pool and is rescued by restoration of progenitor cell function. Circulation 2010; 121(2): 276-292

54. Quaini F, Urbanek K, Beltrami AP, Finato N, Beltrami CA, Nadal-Ginard B, Kajstura J, Leri A, Anversa P. Chimerism of the transplanted heart. N Engl J Med 2002; 346(1): 5-15. 